\title{
El manual de inducción y bienvenida como parte de la cultura organizacional
}

\section{The manual of induction and welcome as part of the organizational culture}

Washington Santillán Marroquín

Lily Merizalde Figueroa

Juan Carlos Flores Díaz

Universidad Internacional SEK Ecuador, Ecuador

Autores para correspondencia:w.santillan@uisek.edu.ec

Fecha de recepción: 04 de Diciembre 2017 - Fecha de aceptación: 15 de Junio de 2018

Resumen: En la era del Talentismo, es necesario como gestores humanos, contar con las herramientas y competencias adecuadas para poder atraer, motivar, retener y potencializar el talento humano dentro de las organizaciones, una forma de realizar esto, es generando una primera "buena impresión" en el nuevo colaborador cuando ingresa a formar parte del equipo de trabajo en una Organización. Un aspecto clave para realizar lo antes mencionado es, resaltar los aspectos fundamentales de la cultura organizacional, generando en el nuevo colaborador: un alto sentido de pertenencia, identificación con los valores, alineación al plan estratégico, misión, visión y los objetivos de la institución y finalmente, compromiso mediante la creación e implementación de una herramienta de inducción y bienvenida, que permita orientarlo al nuevo entorno laboral al que se enfrenta (empresa y puesto de trabajo) y fomentar una rápida adaptación que le permita ser eficiente, productivo y concretar los resultados para lo cual fue contratado. (Santillán 2017)

Palabras Claves: atraer; motivar; retener; potencializar; talento; cultura corporativa; pertenencia; compromiso; inducción; adaptación; eficiente; productivo; resultados

Abstract: In the era of the Talentismo, it is necessary as managers of human talent, have appropriate skills and tools to be able to attract, motivate, retain and empower human talent within organizations, a way to do this, it is generating a first "good impression" on the new contributor when he joined to form part of the team in an organization. A key aspect for the afore-mentioned is, highlight the fundamental aspects of the corporate culture of the Organization, generating new collaborator: a high sense of belonging, identification with the values, alignment to the strategic plan, Mission, Vision and Goals of the institution and ultimately engagement through the creation and implementation of a tool of induction and welcome, which allows to orient it to the new working environment is facing (company and job) and promote a rapid adaptation that allows them to be efficient, productive and concrete outcomes for which he was hired. (Santillan 2017)

Key Words: attract; motivate; retain; promote; talent; corporate culture; belonging; commitment; induction; adaptation; efficient; productive; results 


\section{Introducción}

El nuevo colaborador debe sentirse bien recibido y cómodo, parte del equipo de trabajo y comprender de manera general aspectos claves de la organización a la que ingresa. Es necesario brindar al colaborador las herramientas necesarias para que conozca la manera en cómo la empresa actúa y hace las cosas. Por esta razón, el departamento de talento humano debe brindar una correcta orientación para que pueda desempeñarse con efectividad. En este caso es necesario brindarles una inducción ofreciendo información importante de la Institución y sus actividades, a fin de que se adapten con facilidad a la misma.

Dentro de la cultura corporativa de las organizaciones, que incluyen el área de talento humano la cual enfrenta la realidad intangible pero inevitable de una cultura y fisionomía propia, por lo que implica integrar con eficacia y rapidez a los nuevos miembros que ingresen a la organización. Es por este motivo que en la dinámica del desarrollo del proceso de inducción, se ha evidenciado que un programa de socialización logra su objetivo en la medida que se consigue acelerar la incorporación de nuevos colaboradores en beneficio de las organizaciones. (Werther, 2000, pp.221-225).

Las organizaciones deben ofrecer oportunidades de involucramiento en los colaboradores para que estos puedan desarrollar un sentido de pertenencia y compromiso con la organización y sus actividades. La palabra "pertenecer" se relaciona con compromiso, es decir si el colaborador se apropia de los objetivos y valores organizacionales, su compromiso con la organización será mayor. (Alles, 2008, p.36). Por esta razón el no contar con un proceso de inducción de personal puede desencadenar en falta de compromiso y sentido de pertenencia en los colaboradores.

Por otro lado, la ausencia de una herramienta para optimizar el proceso de inducción o también conocido como socialización, eleva el nivel de ansiedad y tensión que generalmente siente un ser humano al iniciar sus actividades laborales en una nueva organización, el colaborador debe sentirse bien recibido desde su primer día. (Dessler, 2009, p.291).

La gestión del talento humano en las organizaciones centra su atención en el desarrollo de procesos que eleven la satisfacción de sus colaboradores. El proceso de inducción de acuerdo a varios estudios realizados, ha permitido reducir el nivel de ansiedad y tensión que presentan los colaboradores al ingresar a un nuevo ambiente de trabajo, garantizando una adaptación rápida y confortable.

De acuerdo a Werther (2000, p.195) el índice de rotación y compromiso en colaboradores son causas de fenómenos internos de la organización como: su cultura organizacional, política disciplinaria de la organización, beneficios, condiciones físicas de trabajo. Estos fenómenos son expresados en un proceso de inducción.

Teniendo en cuenta este aspecto, es importante desarrollar una herramienta de inducción para el personal que permita informar aspectos relevantes de la institución, así como también incrementar el grado de compromiso y sentido de pertenencia por parte de los colaboradores.

\section{Método}


El presente estudio es un tipo de investigación mixta (cuantitativa y cualitativa) de corte estudio de caso en una Universidad Ecuatoriana dentro de una modalidad para un Proyecto de APLICACIÓN, desarrollo, motivación y retención de talento. Se realizó en una Población de 90 docentes con una muestra específica de 50 docentes. Los instrumentos que se utilizaron fueron los siguientes:

- Cuestionario

- Escala de likert para evaluar 4 criterios del manual de inducción

- Manual de inducción y bienvenida

\section{Levantamiento de datos}

\section{Resultados}

Se estableció que para la elaboración de este proyecto de investigación se encuestaría a 50 docentes de la Universidad que servirá de ejemplo. Los docentes fueron seleccionados por los siguientes criterios: pertenecer a cualquiera de las facultades de la universidad y estar trabajando en la Institución de 0 a 1 año o más en relación de dependencia.

La aplicación de las encuestas se realizó en junio del 2016. Durante el levantamiento de información existió cooperación por parte de los docentes encuestados, permitiendo llevar a cabo el proceso de manera rápida y eficiente.

\section{Presentación y análisis de resultados}

Las encuestas aplicadas a los 50 docentes fueron tabuladas en dos grupos; el primero aquellos docentes que están trabajando en la Institución de 0 a 1 año, el segundo grupo aquellos docentes que trabajan más de un año en la Institución.

El análisis de los resultados permitió evidenciar las diferencias en cuanto a la percepción del proceso de inducción y la necesidad de contar con un manual de inducción y bienvenida basado en la cultura corporativa de la dicha Universidad.

Por otro lado, los resultados determinaron información que debe contener el manual de inducción y bienvenida para docentes.

\begin{tabular}{ccc}
\hline Años en la organización & Respuestas & $\%$ \\
\hline 0 a 1 año & 19 & $38 \%$ \\
1 año o más & 31 & $62 \%$ \\
TOTAL & 50 & $100 \%$ \\
\hline
\end{tabular}




\section{Análisis}

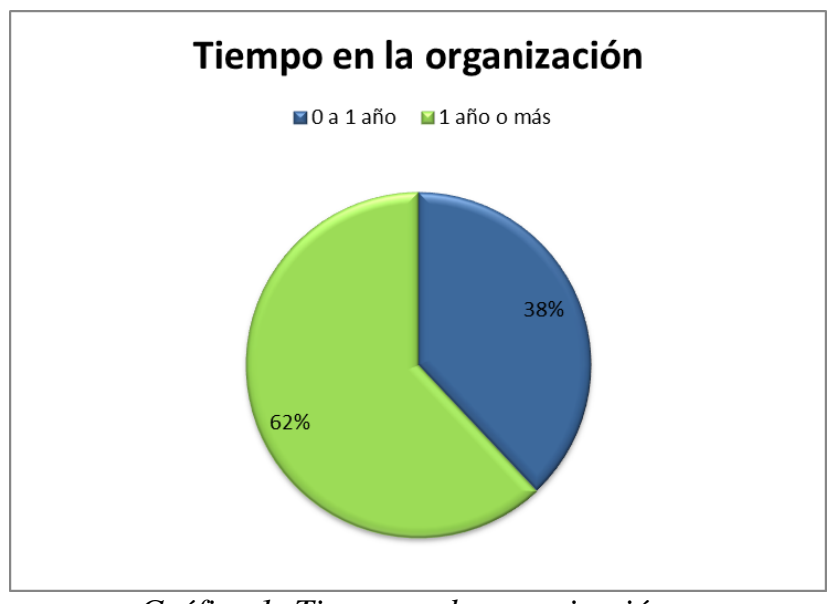

Gráfico 1: Tiempo en la organización

De la muestra seleccionada, el $62 \%$ son docentes que llevan 1 año o más trabajando en la Institución. Por otro lado, el 32\% restante son docentes que trabajan menos de un año en la Universidad. Se puede determinar que la mayoría de los docentes encuestados llevan trabajando en la Universidad más de un año.

Tabla 1: Recibió información y bienvenida (inducción) - Grupo 1

\begin{tabular}{ccc}
\hline Opción & Frecuencia & Porcentaje \\
\hline SI & 11 & $58 \%$ \\
NO & 8 & $42 \%$ \\
TOTAL & 19 & $100 \%$ \\
\hline
\end{tabular}

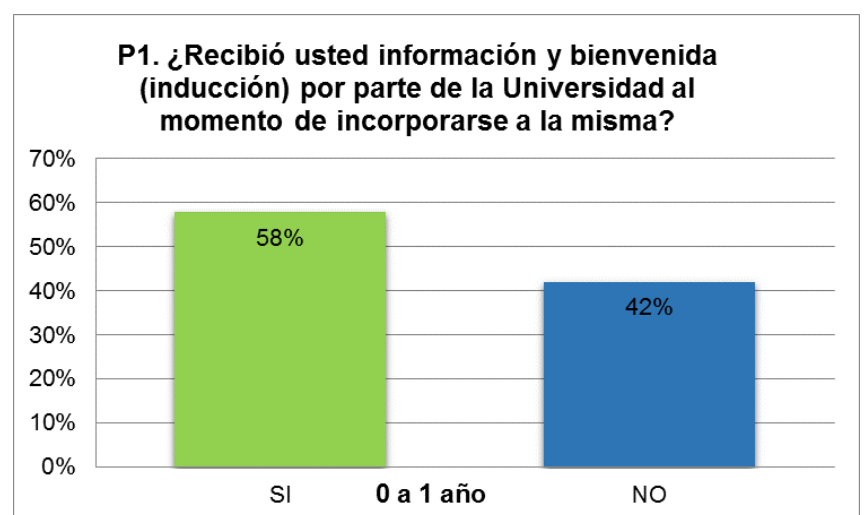

Gráfico 2: Recibió información y bienvenida (inducción) - Grupo 1

Tabla 2: Recibió información y bienvenida (inducción) - Grupo 2

\begin{tabular}{ccr}
\hline Opción & Frecuencia & Porcentaje \\
\hline SI & 3 & $10 \%$ \\
NO & 28 & $90 \%$ \\
TOTAL & 31 & $100 \%$ \\
\hline
\end{tabular}




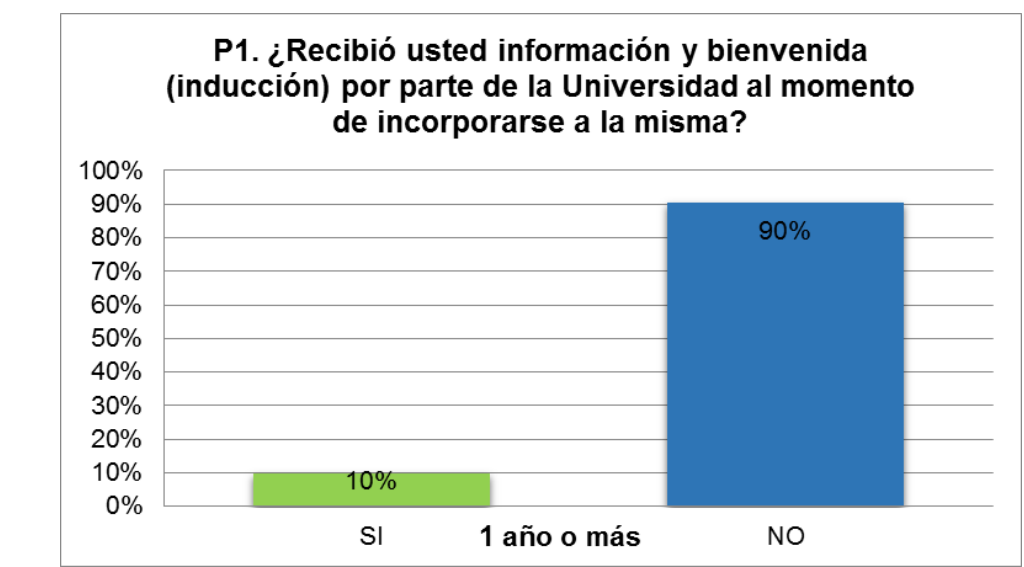

Gráfico 3: Recibió información y bienvenida (inducción) - Grupo 2

\section{Análisis}

En la primera pregunta, en el primer grupo, el 58\% de docentes dicen haber recibido información y bienvenida (inducción) por parte de la universidad al momento de incorporarse a la misma. Por otro lado, en el segundo grupo, el 10\% de docentes afirman si haber recibido información y bienvenida (inducción). Conforme a los resultados obtenidos se puede determinar que talento humano ha iniciado un proceso de inducción y bienvenida para los nuevos docentes este último año, careciendo de este proceso los docentes que tienen más de un año en la organización.

Tabla 3: Cumplió expectativas - Grupo 1

\begin{tabular}{ccc}
\hline Opción & Frecuencia & Porcentaje \\
\hline SI & 5 & $45 \%$ \\
NO & 6 & $55 \%$ \\
TOTAL & 11 & $100 \%$ \\
\hline
\end{tabular}

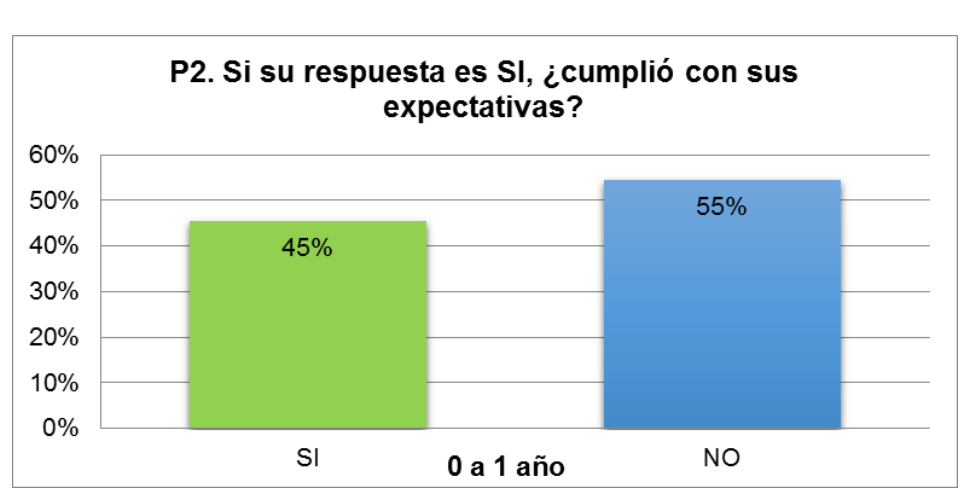

Gráfico 4: Cumplió expectativas - Grupo 1

Tabla 4: Cumplió expectativas - Grupo 1

\begin{tabular}{ccc}
\hline Opción & Frecuencia & Porcentaje \\
\hline SI & 2 & $67 \%$ \\
NO & 1 & $33 \%$ \\
\hline
\end{tabular}




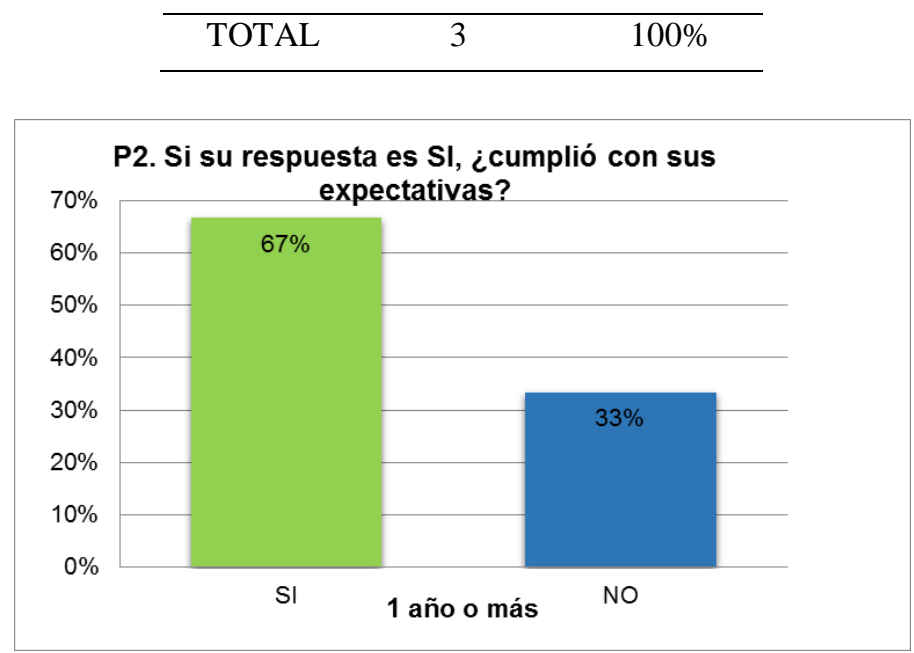

Gráfico 5: Cumplió expectativas - Grupo 2

\section{Análisis}

En la pregunta 2, de aquellos que contestaron que sí recibieron inducción por parte de la universidad; del primer grupo el 55\% de docentes contestaron que esta inducción no cumplió con sus expectativas. Por otro lado, en el segundo grupo, de docentes que si recibieron la inducción, un 33\% aseguró que ésta no cumplió con sus expectativas.

Se puede determinar que los docentes nuevos y como aquellos que tienen más de un año mencionan que no se cumplió con el nivel de expectativa requerido por ellos al momento de integrarse a la universidad.

Tabla 5: Es necesario inducción a docentes - Grupo 1

\begin{tabular}{ccc}
\hline Opción & Frecuencia & Porcentaje \\
\hline SI & 8 & $100 \%$ \\
NO & 0 & $0 \%$ \\
TOTAL & 8 & $100 \%$ \\
\hline
\end{tabular}

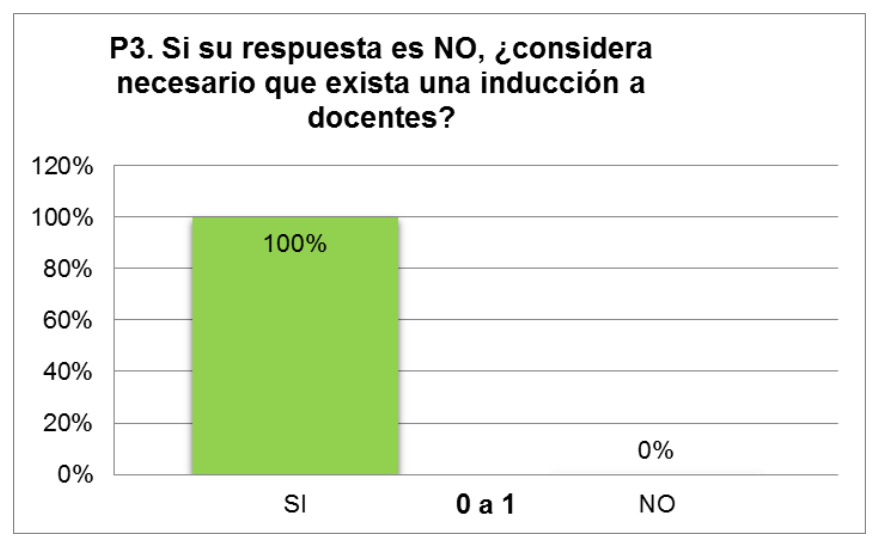

Gráfico 6: Es necesario inducción a docentes - Grupo 1

Tabla 6: Es necesario inducción a docentes - Grupo 2 


\begin{tabular}{ccc}
\hline Opción & Frecuencia & Porcentaje \\
\hline SI & 28 & $100 \%$ \\
NO & 0 & $0 \%$ \\
TOTAL & 28 & $100 \%$ \\
\hline
\end{tabular}

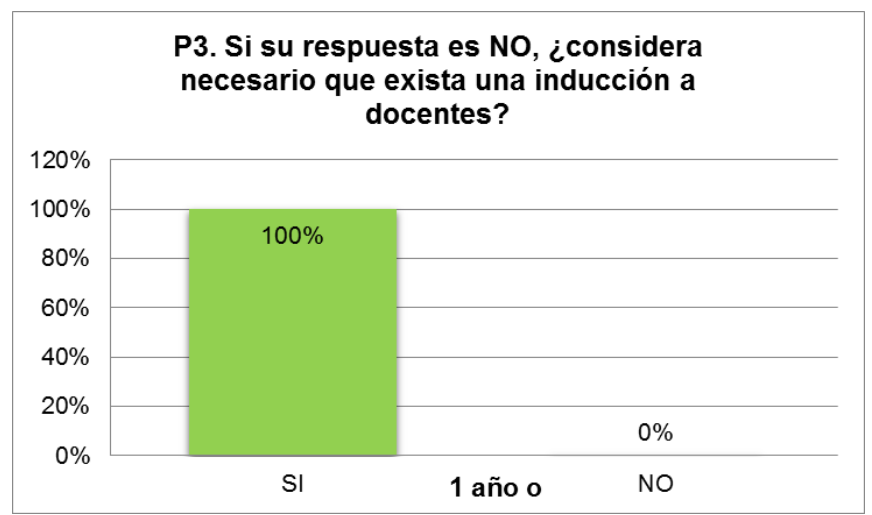

Gráfico 7: Es necesario inducción a docentes - Grupo 2

\section{Análisis}

En la pregunta 3, de aquellos docentes que aseguraron no haber recibido inducción al ingresar a la Universidad; en ambos grupos se obtuvo como resultado que el $100 \%$ de docentes consideran necesario la existencia de una inducción para ellos por parte de la universidad. Como conclusión se podría decir que, para los docentes, el conocer aspectos sobre la universidad y su actividad a realizar dentro de ella, es importante y necesaria.

Tabla 7: Información que un docente debe conocer al incorporarse a la Universidad - Grupo 1 Opción

Valores, misión, visión, organigrama

Horario de trabajo y beneficios Frecuencia Porcentaje

Antecedentes de la universidad

Sus principales departamentos y actividades

\begin{tabular}{cc}
7 & $17 \%$ \\
10 & $24 \%$ \\
1 & $2 \%$ \\
8 & $19 \%$ \\
& \\
7 & $17 \%$ \\
9 & $21 \%$ \\
42 & $100 \%$ \\
\hline
\end{tabular}

Instalaciones

Todas las anteriores

$21 \%$

TOTAL

P4. ¿En base a su experiencia, qué considera usted como docente importante conocer al momento de incorporarse a la Universidad? Puede elegir varias opciones

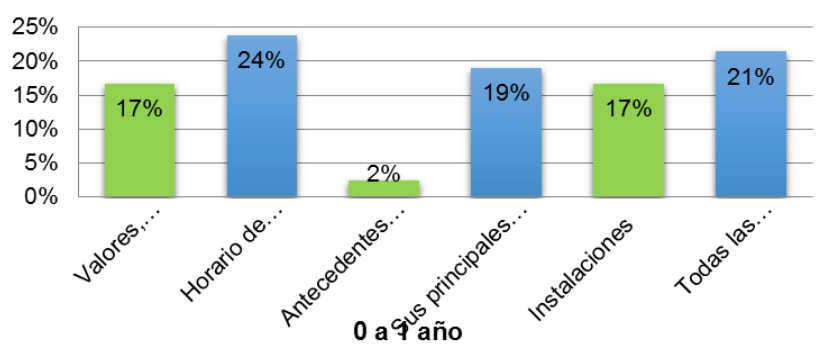

Gráfico 8: Información que un docente debe conocer al incorporarse a la Universidad-Grupo 1 
Tabla 8: Información que un docente debe conocer al incorporarse a la Universidad - Grupo 2

\begin{tabular}{lcc}
\hline \multicolumn{1}{c}{ Opción } & Frecuencia & Porcentaje \\
\hline Valores, misión, visión, organigrama & 12 & $19 \%$ \\
Horario de trabajo y beneficios & 13 & $21 \%$ \\
Antecedentes de la universidad & 3 & $5 \%$ \\
Sus principales departamentos y actividades & 9 & $14 \%$ \\
& & \\
Instalaciones & 10 & $16 \%$ \\
Todas las anteriores & 16 & $25 \%$ \\
TOTAL & 63 & $100 \%$ \\
\hline
\end{tabular}

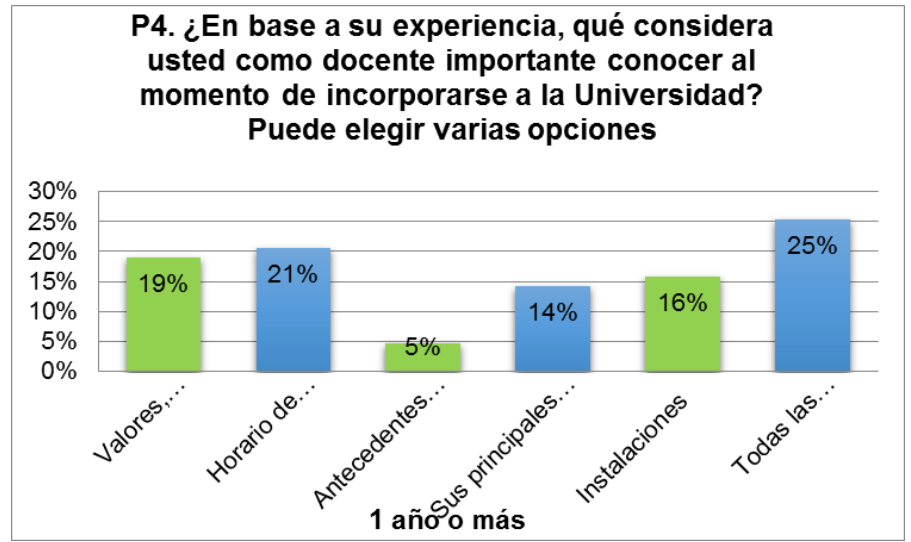

Gráfico 9: Información que un docente debe conocer al incorporarse a la Universidad - Grupo 2

\section{Análisis}

En la pregunta 4, se realizó la tabulación por frecuencia de los indicadores y no por encuestado. El primer grupo hizo énfasis con un $21 \%$ en que todos los aspectos enumerados en la encuesta son importantes dar a conocer a los docentes, sin embargo, la opción de conocer el horario de trabajo y beneficios tuvo un $24 \%$ de importancia. El $19 \%$ de docentes dijo que deben conocer desde el inicio de sus actividades en la universidad, los principales departamentos de la misma. Un $17 \%$ de docentes asegura que la misión, visión, valores y organigrama, así como las instalaciones de la universidad se deben dar a conocer a los docentes. Un $2 \%$ de docentes mencionaron que conocer aspectos históricos de la universidad es de gran importancia.

Por otro lado, en el segundo grupo de docentes, aseguraron con un 25\% que todas las opciones de la encuesta son importantes conocer al momento de incorporarse a la universidad. Horario de trabajo $21 \%$, Valores, misión, visión y organigrama $19 \%$, instalaciones $16 \%$, sus principales departamentos 14\%, antecedentes históricos 5\%.

Como conclusión se podría decir que la mayoría de docentes considera de suma importancia conocer lo que incluye un manual de inducción y bienvenida (valores, misión, visión, organigrama, horario de trabajo, instalaciones, principales departamentos y antecedentes de la universidad).

Tabla 9: Requerimientos frecuentes por los docentes encuestados - Ambos grupos Aspectos relevantes propuestos por los docentes encuestados

\begin{tabular}{lll}
\hline Requerimientos frecuentes de docentes encuestados & Frecuencia & $\%$ \\
\hline
\end{tabular}




\begin{tabular}{ccc}
\hline Reglamentos & 3 & $43 \%$ \\
Manual Seguridad y salud ocupacional & 2 & $29 \%$ \\
Aspectos específicos de donde se va a trabajar & 2 & $29 \%$ \\
TOTAL & 7 & $100 \%$
\end{tabular}

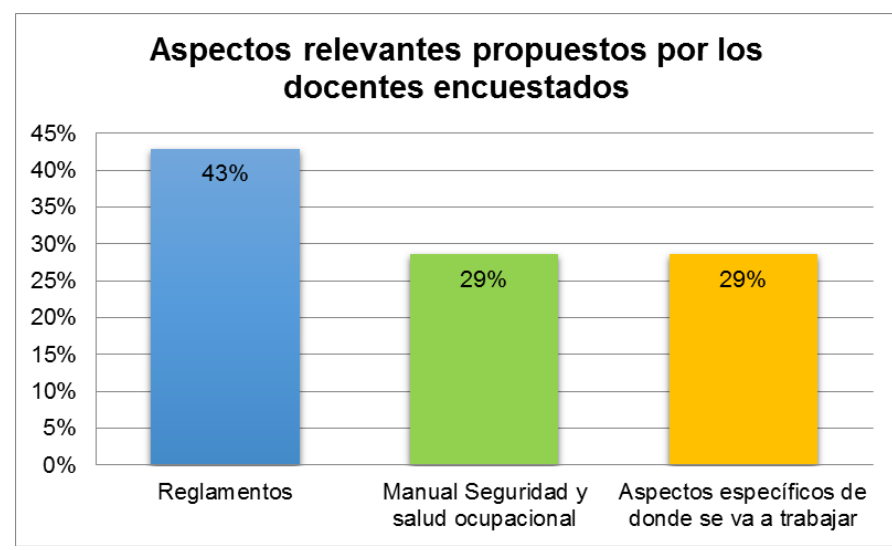

Gráfico 10: Requerimientos frecuentes por los docentes encuestados - Ambos grupos

\section{Análisis}

Como parte de la pregunta 4, los encuestados de ambos grupos sugieren los siguientes aspectos para incorporar al manual de inducción: reglamentos $43 \%$, manual de seguridad y salud ocupacional $29 \%$ y aspectos específicos de donde se va a trabajar $29 \%$. Estos aspectos fueron analizados por su frecuencia, es decir fueron los que más se repitieron.

Considerando el interés presentado por los encuestados es necesario que el nuevo manual de inducción y bienvenida para docentes de la Universidad, incorpore lo requerido por los mismos. Sin embargo, en el tema de aspectos específicos de donde se va a trabajar, se incluirán en el manual únicamente aspectos genéricos ya que en el proceso de inducción los docentes reciben información de sus actividades específicas.

Tabla 10: Documentar y difundir la cultura corporativa a docentes - Grupo 1

\begin{tabular}{ccc}
\hline Opción & Frecuencia & Porcentaje \\
\hline SI & 18 & $95 \%$ \\
NO & 1 & $5 \%$ \\
TOTAL & 19 & $100 \%$ \\
\hline
\end{tabular}




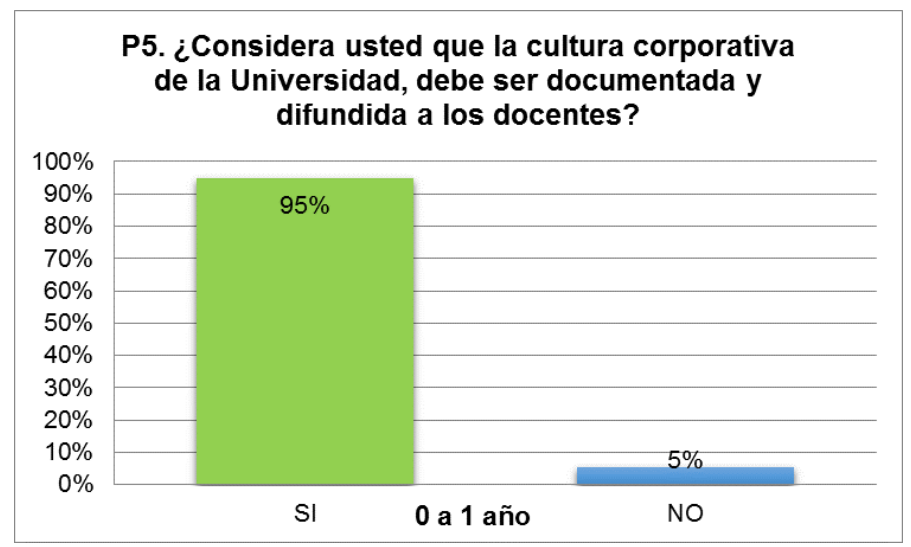

Gráfico 11: Documentar y difundir la cultura corporativa a docentes - Grupo 1

Tabla 11: Documentar y difundir la cultura corporativa a docentes - Grupo 2

\begin{tabular}{ccc}
\hline Opción & Frecuencia & Porcentaje \\
\hline SI & 31 & $100 \%$ \\
NO & 0 & $0 \%$ \\
TOTAL & 31 & $100 \%$ \\
\hline
\end{tabular}

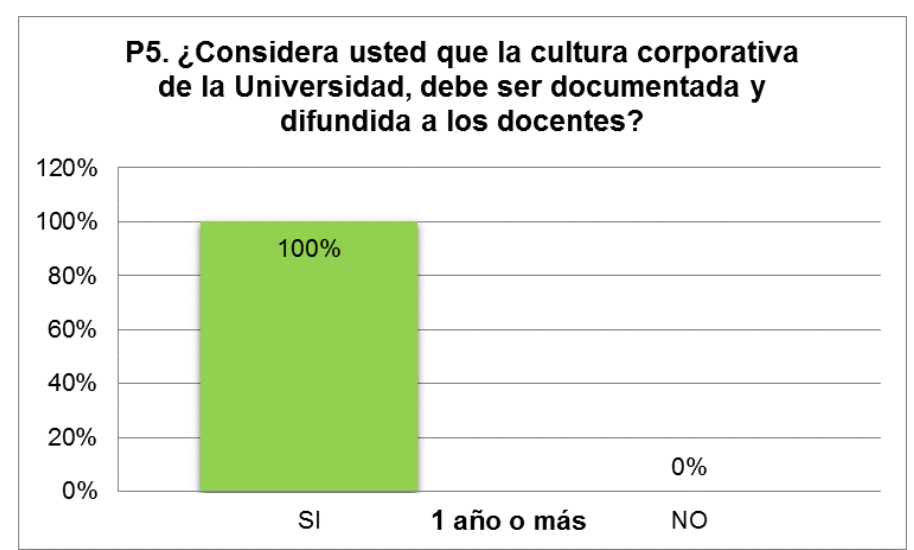

Gráfico 12: Documentar y difundir la cultura corporativa a docentes - Grupo 2

\section{Análisis}

En la pregunta 5, un 95\% de docentes del primer grupo considera que la cultura corporativa debe ser documentada y difundida. Sin embargo, en el segundo grupo, la unanimidad se hizo presente en esta pregunta, obteniendo un $100 \%$ en el SI. Se podría decir que, en su gran mayoría de docentes de la universidad, consideran que deberían conocer la cultura corporativa de la Institución, la misma que debe ser documentada y difundida.

Tabla 12: Deber suministrarse un manual de inducción y bienvenida a docentes - Grupo 1

\begin{tabular}{ccc}
\hline Opción & Frecuencia & Porcentaje \\
\hline SI & 19 & $100 \%$ \\
NO & 0 & $0 \%$ \\
TOTAL & 19 & $100 \%$ \\
\hline
\end{tabular}




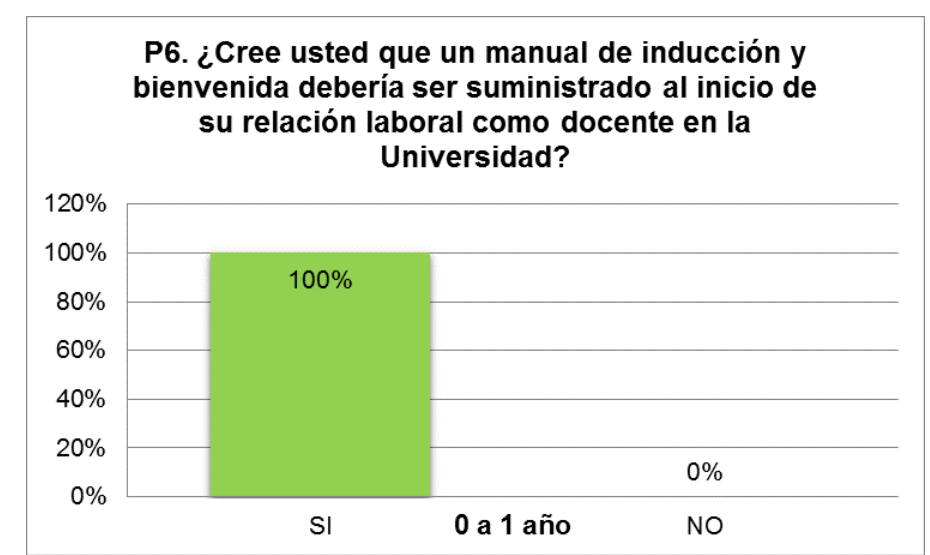

Gráfico 13: Deber suministrarse un manual de inducción y bienvenida a docentes - Grupo 1

Tabla 13: Deber suministrarse un manual de inducción y bienvenida a docentes - Grupo 2

\begin{tabular}{ccc}
\hline Opción & Frecuencia & Porcentaje \\
\hline SI & 31 & $100 \%$ \\
NO & 0 & $0 \%$ \\
TOTAL & 31 & $100 \%$ \\
\hline
\end{tabular}

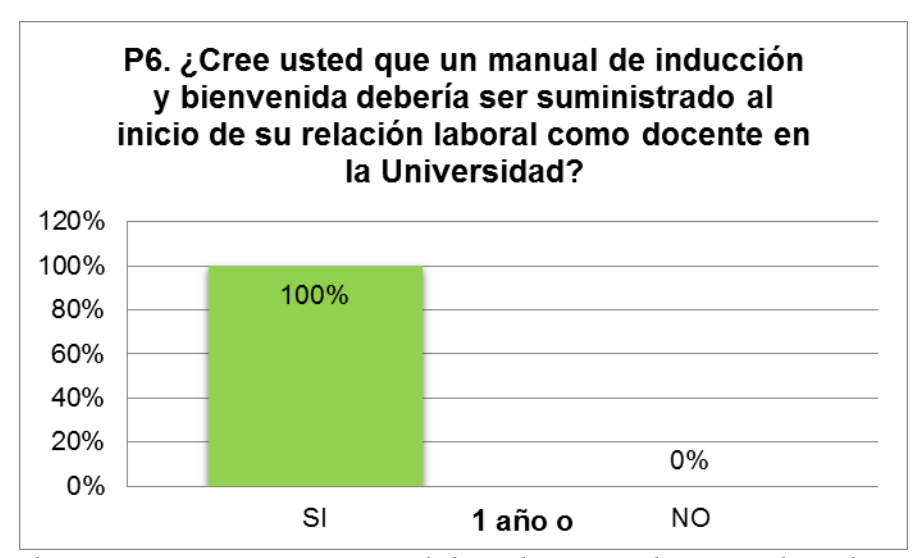

Gráfico 14: Deber suministrarse un manual de inducción y bienvenida a docentes - Grupo 2

\section{Análisis}

En la pregunta 6, en ambos grupos se evidenció un 100\%, de que un manual de inducción y bienvenida debe ser suministrado a los docentes al inicio de su relación laboral con la Universidad. Como conclusión se podría decir que la elaboración de un manual de inducción y bienvenida para los docentes sería de gran utilidad para los mismos, permitiendo cumplir sus expectativas en el proceso de socialización - inducción. 
Esquema del manual de inducción y bienvenida

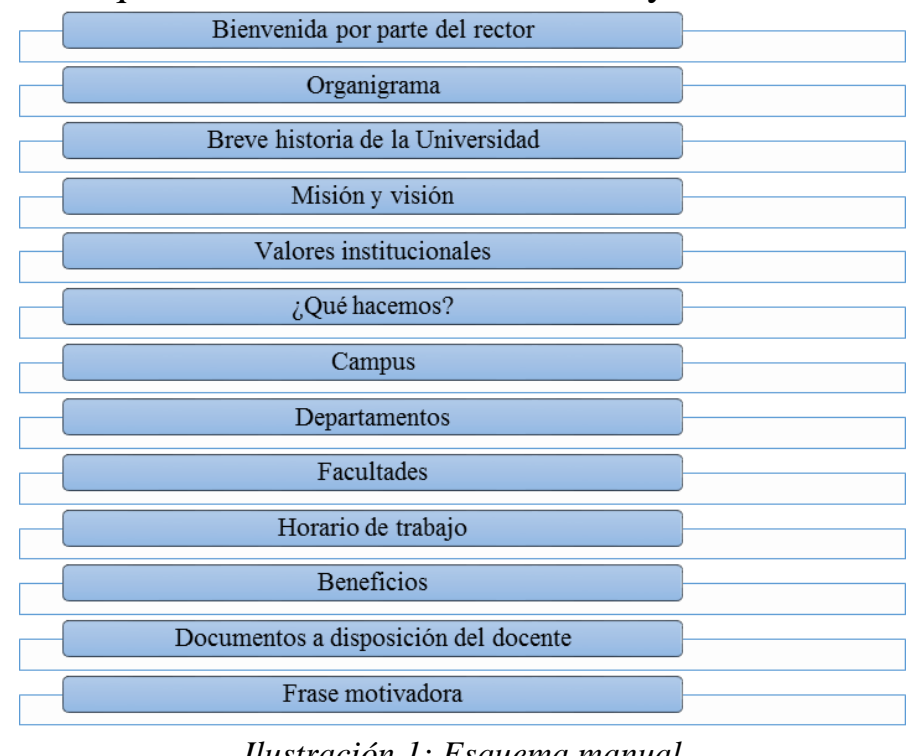

Ilustración 1: Esquema manual

Validación del manual de inducción por expertos

Tabla 14: Relevancia del contenido

\begin{tabular}{|l|c|c|}
\hline \multicolumn{1}{|c|}{ Opción } & Frecuencia & \% \\
\hline $\begin{array}{l}\text { Totalmente de } \\
\text { acuerdo }\end{array}$ & 4 & $100 \%$ \\
\hline De acuerdo & 0 & $0 \%$ \\
\hline $\begin{array}{l}\text { Ni de acuerdo } \\
\text { ni desacuerdo }\end{array}$ & 0 & $0 \%$ \\
\hline En desacuerdo & 0 & $0 \%$ \\
\hline $\begin{array}{l}\text { Totalmente en } \\
\text { desacuerdo }\end{array}$ & 0 & $0 \%$ \\
\hline \multicolumn{1}{|c|}{ TOTAL } & 4 & $100 \%$ \\
\hline
\end{tabular}

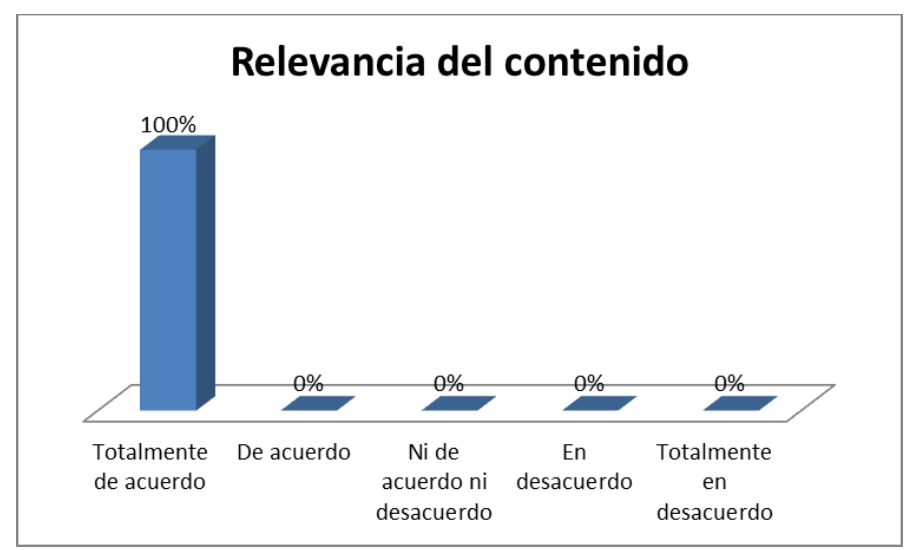

Gráfico 15: Relevancia del contenido

\section{Análisis}

Revista de la Universidad Internacional del Ecuador. URL: https://www.uide.edu.ec/ 
En el primer criterio, el $100 \%$ de expertos opinaron estar totalmente de acuerdo con el contenido presentado en el manual. Como conclusión se puede decir que el contenido del manual de inducción y bienvenida para docentes de la Universidad, es adecuado y relevante.

Tabla 15: Pertinencia del contenido

\begin{tabular}{lll}
\hline Opción & Frecuencia & $\%$ \\
\hline Totalmente de acuerdo & 4 & $100 \%$ \\
De acuerdo & 0 & $0 \%$ \\
Ni de acuerdo ni desacuerdo & 0 & $0 \%$ \\
En desacuerdo & 0 & $0 \%$ \\
Totalmente en desacuerdo & 0 & $0 \%$ \\
TOTAL & 4 & $100 \%$ \\
\hline
\end{tabular}

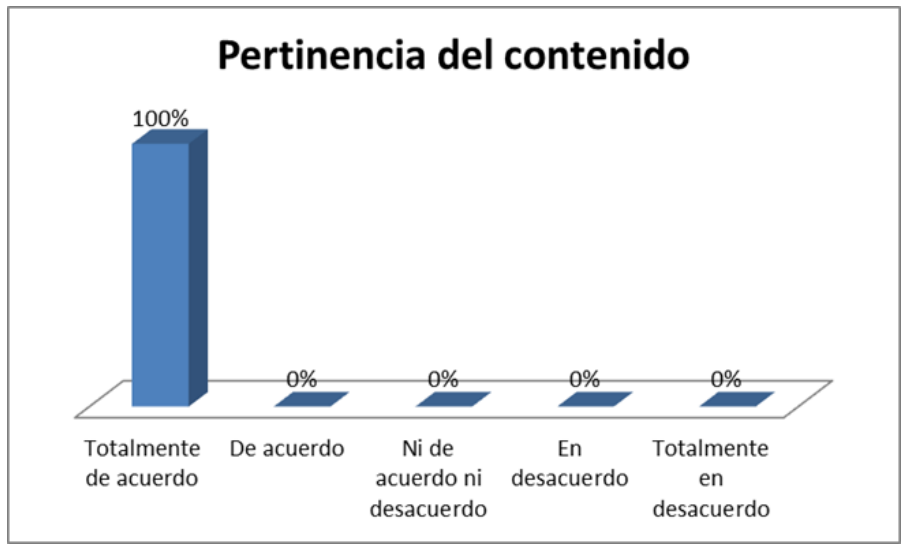

Análisis

Gráfico 16: Pertinencia del contenido

En el segundo criterio, el $100 \%$ de expertos opinaron estar totalmente de acuerdo con que el contenido presentado en el manual es pertinente. Como conclusión se puede decir que el contenido del manual de inducción y bienvenida para docentes de la Universidad, tiene un grado alto de pertinencia.

Tabla 16: Utilidad del manual

\begin{tabular}{lll}
\hline Opción & Frecuencia & $\%$ \\
\hline Totalmente de acuerdo & 4 & $100 \%$ \\
De acuerdo & 0 & $0 \%$ \\
Ni de acuerdo ni & 0 & $0 \%$ \\
desacuerdo & & \\
En desacuerdo & 0 & $0 \%$ \\
Totalmente en desacuerdo & 0 & $0 \%$ \\
TOTAL & 4 & $100 \%$ \\
\hline
\end{tabular}




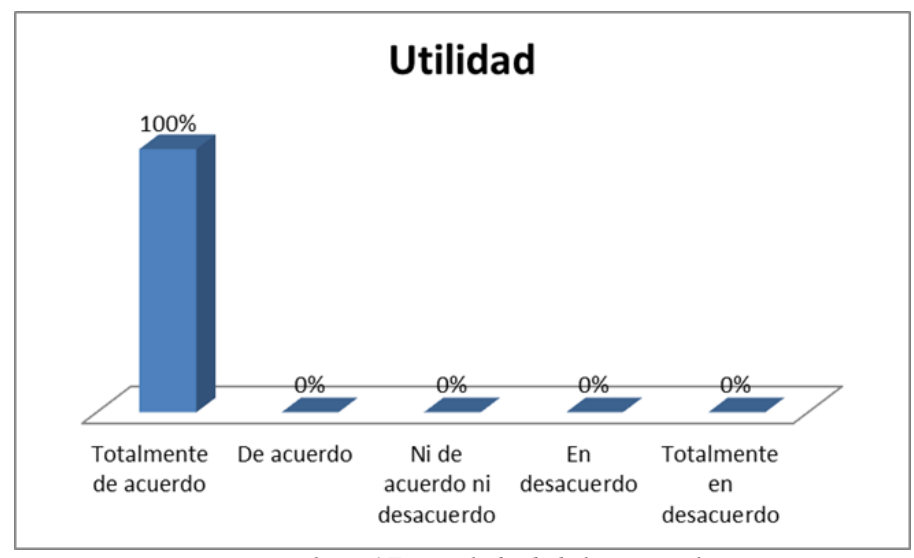

Gráfico 17: Utilidad del manual

\section{Análisis}

En el tercer criterio, el $100 \%$ de expertos opinaron estar totalmente de acuerdo con la utilidad del manual de inducción y bienvenida. Como conclusión se puede decir que el manual de inducción y bienvenida para docentes de esta Universidad, es de gran utilidad para el proceso de inducción.

Tabla 17: Transmite cultura corporativa

\begin{tabular}{|l|c|c|}
\hline \multicolumn{1}{|c|}{ Opción } & Frecuencia & $\%$ \\
\hline $\begin{array}{l}\text { Totalmente de } \\
\text { acuerdo }\end{array}$ & 4 & $100 \%$ \\
\hline De acuerdo & 0 & $0 \%$ \\
\hline $\begin{array}{l}\text { Ni de acuerdo } \\
\text { ni desacuerdo }\end{array}$ & 0 & $0 \%$ \\
\hline En desacuerdo & 0 & $0 \%$ \\
\hline $\begin{array}{l}\text { Totalmente en } \\
\text { desacuerdo }\end{array}$ & 0 & $0 \%$ \\
\hline \multicolumn{1}{|c|}{ TOTAL } & 4 & $100 \%$ \\
\hline
\end{tabular}

\section{Análisis}

En el cuarto y último criterio, el 100\% de expertos opinaron estar totalmente de acuerdo con que el manual de inducción y bienvenida transmite la cultura corporativa de la Universidad y la información necesaria para la gestión de docentes. Como conclusión se puede decir que el manual de inducción y bienvenida elaborado transmite la cultura corporativa de la Universidad a través de información importante para que el docente desempeñe sus actividades.

\section{Aplicación práctica}

La aplicación práctica de este proyecto de investigación es la elaboración de un manual de inducción y bienvenida para docentes de la Universidad basado en su cultura corporativa. Este manual es una herramienta que facilitará y optimizará el proceso de inducción en la Universidad. 


\section{Conclusiones}

Existe diferente percepción en los docentes sobre el proceso de inducción, ya que señalan que los antecedentes históricos de la universidad no son relevantes conocer, sin embargo, manifiestan que toda la cultura corporativa de la Universidad es importante para ellos.

De acuerdo a las respuestas obtenidas a los docentes encuestados, el manual de inducción y bienvenida deberá ser debidamente documentado y socializado al momento de su incorporación a la Universidad, en vista que los docentes manifiestan predisposición para ser parte de un proceso de inducción.

Es necesario diseñar una herramienta que permita informar a los nuevos docentes aspectos claves de la universidad, y que a su vez exprese la cultura e identidad corporativa de la Universidad bajo los esquemas básicos que contiene un manual de inducción y bienvenida.

El proceso de inducción actual llevado a cabo por talento humano de la Universidad, de acuerdo a la investigación los encuestados en su mayoría manifestaron que la misma no cumple con las expectativas requeridas por ellos, en especial los docentes que tienen más de un año en dependencia laboral.

Los docentes encuestados manifiestan que debe incorporarse al manual de inducción y bienvenida, los siguientes aspectos: reglamentos, manual de salud y seguridad ocupacional y aspectos específicos de donde se va a trabajar.

Por medio de este proyecto de investigación se llegó a la conclusión de que la elaboración de un manual de inducción y bienvenida para docentes de la Universidad en el que se realizó este estudio, es factible y permitirá fomentar un sentido de pertenencia y empoderamiento de su cultura corporativa. Los expertos que participaron en la revisión del manual de inducción y bienvenida para los docentes de la Universidad, validaron que el contenido del manual tiene relevancia, a 


\section{Bibliografía}

Alles, M. (2008). Comportamiento organizacional. Argentina: Ediciones Garnica. 36,37,54,58.

Ariza, J., Morales, A., Morales, E. (2004). Dirección y administración integrada de personas. Fundamentos, procesos y técnicas en práctica. Madrid: Mc Graw Hill. 119.

Avesca. (s/a). Manual de inducción y bienvenida.

Barraza, A., Acosta, M. (2008). Compromiso organizacional en docentes de una institución de educación media superior. México: Vol. 8. 22-28. Recuperado de: (consultado el 09 de junio de 2016). http://www.redalyc.org/pdf/1794/179420818003.pdf

Chiavenato, I. (2000). Administración de Recursos Humanos. Bogotá: Quinta edición. Mc Graw Hill. 108-285

Chiavenato, I. (2011). Planeación estratégica. Fundamentos y aplicaciones. México: Segunda edición. Mc Graw Hill. 72-80

Constitución de la República del Ecuador. (2008).

Cuesta, A. (2010). Gestión del talento humano y del conocimiento. Bogotá: Primera edición. Ecoe ediciones. 47-299

Dessler, G. (2001). Administración de personal. México: Pearson Educación. 249. Recuperado de: (consultado el día 1 de junio 2016)

https://books.google.com.ec/books?id=00dKezzNE-AC\&dq=induccion+de+personal++ dessler\&hl=es\&source $=$ gbs_navlinks_s

Dessler, G. (2009). Administración de Recursos Humanos. México: Décimo quinta edición. Pearson educación. 291-293

García, S., Del Val, M. (1993). Cultura corporativa y competitividad de la empresa española. Ediciones Díaz de Santos. 31-33. Recuperado de: (consultado el día 1 de junio 2016) https://books.google.com.ec/books?id=ncctOr0HKwQC\&dq=cultura+corporativo\&hl=e s\&source=gbs_navlinks_s

Koontz, H., Weihrich, H., Cannice, M. (2008). Administración: Una perspectiva global y empresarial. México: Décimo tercera edición. Mc Graw Hill. 135-335

Lessem, R. (1992). Gestión de la cultura corporativa. Madrid: Ediciones Díaz de Santos. 12. Recuperado de: (consultado el día 2 de junio 2016) https://books.google.com.ec/books?id=4xdxz4tzAgwC\&dq=cultura+corporativ\&hl=es \&source=gbs_navlinks_s 
Ley Orgánica de Educación Superior. (2010).

Medina, J. (2009). Pensar, planificar, ejecutar y evaluar estratégicamente. Primera edición. Codeu editorial. 23-29

Meighan, M. (1992). Programas de inducción. Entrenamiento, diseño y ejecución. Colombia: Legis editores. 4-69

Pintado, M. (2013). Imagen corporativa: influencia en la gestión empresarial. Madrid: ESIC Editorial. 13-14. Recuperado de: (consultado el día 1 de junio 2016) https://books.google.com.ec/books?id=x98cgeMFA30C\&dq=cultura+corporativo \&hl=e s\&source=gbs_navlinks_s

Plan Nacional del Buen Vivir. (2013-2017).

Pontificia Universidad Católica del Ecuador. (s/a). Manual de bienvenida PUCE. Recuperado de: (consultado el día 3 de junio 2016) http://www.puce.edu.ec/intranet/documentos/ManualBienvenida-Docentes-PUCE.pdf

Sánchez, H. (2010). Administración educativa y liderazgo, manual. Cuenca: Universidad Politécnica Salesiana. 273-274

Sanz, M., González, M. (2005). Identidad corporativa: Claves de la comunicación empresarial. Madrid: ESIC Editorial. 18-22. Recuperado de: (consultado el día 1 de junio 2016) https://books.google.com.ec/books?id=dgDXUwBDd60C\&dq=cultura+corporativ\&hl= es\&source=gbs_navlinks_s

Sampieri, R., Fernández, C., Baptista, P. (2006). Metodología de la investigación. México: Mc Graw Hill. 99 - 269

Santillán,W.(2017). Nuevas prácticas para mejorar el clima laboral. Quito: Don Bosco.

Thompson, A., Strickland, A., Gamble, J. (2008). Administración estratégica. Teoría y casos. México: Décimo quinta edición. Mc Graw Hill. 415-417

Tecnológico Nacional de México. (s/a). Manual de inducción para el personal docente de nuevo ingreso. Recuperado de: (consultado el día 1 de junio 2016) http://www.itcj.edu.mx/SGCdocumentos/Manual\%20de\%20Induccion.pdf

UNESCO. (s/a). Nomenclatura para los campos de las ciencias y la tecnología. Recuperado de: https://upct.es/contenido/doctorado/Documentos/2012/CODIGOS_UNESCO.pdf

Universidad Iberoamericana. (s/a). Manual de inducción. Recuperado de: (consultado el día 3 de junio 2016) http://rh.uia.mx:8080/jsp/acceso/pdf/manual_induccion.pdf 
Universidad Tecnológica de la Selva. (s/a). Manual de Inducción. Recuperado de: (consultado el día 3 de junio 2016) http://www.utselva.edu.mx/transparencia/manual_de_induccion.pdf

Universidad de las Américas. (s/a). Manual de inducción. Recuperado de: (consultado el día 3 de junio 2016) http://www.udla.edu.ec/wp-content/uploads/2015/07/Guia-docente.pdf

Wallance, A. (1963). Cultura y personalidad. Buenos Aires: Editorial Paidós. 15 - 41

Werther, W., Keith, D. (2000). Administración de Personal y Recursos Humanos. México: Quinta edición. Mc Graw Hill. 48-225 